\title{
Rheological, Mechanical and Tribological Properties of Carbon-nanofibre Reinforced Poly (ether ether ketone) Composites
}

\author{
Volker Altstaedt \\ Universitaet Bayreuth, Lehrstuhl fuer Polymere Werkstoffe, Germany \\ Philipp Werner \\ Lehmann \& Voss \& Co, Germany
}

\author{
Jan Sandler \\ University of Cambridge, Department of Materials Science and Metallurgy, UK
}

\begin{abstract}
Poly(ether ether ketone) nanocomposites containing vapour-grown carbon nanofibres (CNF) were produced using standard polymer processing techniques. At high shear rates no significant increase in resin viscosity was observed. Nevertheless, the addition of the CNFs results in a higher melt strength at $360^{\circ} \mathrm{C}$. Electron microscopy confirmed the homogeneous dispersion and alignment of nanofibres in the polymer matrix. Evaluation of the mechanical composite properties revealed a linear increase in tensile stiffness and strength with nanofibre loading fractions up to $15 \mathrm{wt} \% \mathrm{whilst}$ matrix ductility was maintained up to $10 \mathrm{wt} \%$. An interpretation of the composite performance by short-fibre theory resulted in rather low intrinsic stiffness properties of the vapour-grown CNF. Differential scanning calorimetry was used to investigate crystallization kinetics and degree of crystallinity. The CNFs were found not to act as nucleating sites. Furthermore, unidirectional sliding tests against two different counterpart materials (100 Cr6 martensitic bearing steel, X5CrNi18-10 austenitic stainless steel) were performed. The carbon nanofibres were found to reduce the wear rate of PEEK significantly.
\end{abstract}

Keywords: Carbon nanofibres, PEEK, rheology, tribology, discontinuous reinforcements.

\section{Introduction}

Carbon fibres are widely used today as reinforcements for polymer matrices in many high-technology applications on account of their high specific tensile modulus, strength and excellent electrical and thermal properties. There are, in fact, several types of carbon fibres, each with its own structure and resulting properties. Carbon nanotubes and carbon nanofibres (CNF) are of great scientific interest since the properties of a material become increasingly size dependent at low dimensions. Initial theoretical predictions ${ }^{[1,2]}$ and experimental observations ${ }^{[3-6]}$ of exceptional mechanical performance have led to considerable interest in the fabrication of novel polymer nanocomposites containing nanotubes. (For a comprehensive review of carbon nanotubes and their composites see Ref. ${ }^{[7]}$.) A further advantage of such nanoscale reinforcements is an improved processability and recyclability of thermoplastic nanocomposites. Standard polymer processing and recycling techniques are not expected to break down the filler, an issue commonly encountered even in short-fibre reinforced polymers. Furthermore, the small size of the nanofillers ensures an excellent surface finish and can enable reinforcement of fine structures such as fibres, films, and the matrices of conventional composites.

Twelve years after the discovery of carbon nanotubes ${ }^{[8]}$ we still lack a fundamental understanding of the structureproperty relationships for the vast number of different nanostructures available today. It is known that structural differences and defects present in most nanostructures influence their properties ${ }^{[9]}$. To fully exploit their potential, a fundamental understanding of these structure-property relationships is essential.

Polymer nanocomposites enable both an evaluation of the fundamental properties of the reinforcing nanophase and the development of novel composites with improved physical, mechanical and tribological properties.

An ability to predict nanocomposite properties for a given filler type and loading fraction, as has been achieved for long and short carbon fibre reinforced polymers, is desired. Uniform dispersion within the polymer-matrix and improved nanotube-matrix wetting and adhesion are critical issues in the processing of nanocomposites. Tailoring composite properties to a given application also involves

Autor para Correspondência: Volker Altstaedt, Universitaet Bayreuth, Lehrstuhl fuer Polymere Werkstoffe, Universitaetsstr. 30 , 95440 Bayreuth, Germany E-mail: altstaedt@uni-bayreuth.de 
aligning the nanoscale filler to the direction of expected stresses. Furthermore, potential changes in the polymer morphology due to the presence of a nanophase are important to understand and correctly interpret nanocomposites properties.

The use of nanoscale $\mathrm{Si}_{3} \mathrm{~N}_{4}$ - and $\mathrm{SiO}_{2}$-reinforcements in PEEK composites for tribological applications has already produced promising results ${ }^{[10,11]}$. For both nanocomposites the formation of a thin and uniform tenacious transfer film was believed to lead to the observed lower coefficient of friction and the reduced wear rate. Similarly, the use of carbon nanotubes in a copper matrix significantly reduced the coefficient of friction and the wear rate of such nanocomposite ${ }^{[12]}$. Here the nanotubes were observed to act as a lubricant.

The presented work was aimed at using standard polymer processing techniques to produce nanocomposites of vapourgrown carbon nanofibres (CNF) and a semi-crystalline poly(ether ether ketone) (PEEK) matrix, with regard to the resulting mechanical and tribological properties. Particular emphasis was placed on the nanofibre-matrix interaction during crystallisation to allow a fuller understanding of the composite properties.

\section{Experimental}

PEEK powder grade Victrex 450G was purchased from ICI. The vapour-grown CNF were obtained from Applied Sciences Inc, USA. Macroscopic PEEK nanocomposite masterbatches containing $0,5,10$ and $15 \mathrm{wt} \% \mathrm{CNF}$ were prepared using a Berstorff co-rotating twin-screw extruder with a length-to-diameter ratio of 33 . The processing temperatures were set at about $380^{\circ} \mathrm{C}$. Tensile bars according to the ISO 179A standard were manufactured and heat-treated for $30 \mathrm{~min}$ at $200{ }^{\circ} \mathrm{C}$ and 2 hours at $220^{\circ} \mathrm{C}$ prior to testing.

For standard shear viscosity measurements a melt pump with attached capillary die was fed with a single screw extruder to ensure the same thermal history throughout the whole measurement. Three capillary die with different 1/d-ratios where used for measurements at $360{ }^{\circ} \mathrm{C}$. The data was corrected according to Bagley.

Differential scanning calorimetry (DSC) analysis was performed using Perkin Elmer equipment, on samples taken from the core of the heat-treated injection moulded specimens. Crystallisation and melting patterns were recorded at $10{ }^{\circ} \mathrm{C} / \mathrm{min}$ between 50 and $400{ }^{\circ} \mathrm{C}$. In addition, isothermal thermograms were recorded at $303{ }^{\circ} \mathrm{C}$. The samples were quenched after a 5 minutes isothermal period from the molten state at $400{ }^{\circ} \mathrm{C} / \mathrm{min}$ to the chosen temperature.

Macroscopic tensile tests were performed at room temperature with a Zwick universal testing machine. The cross-head speed was set to $0.5 \mathrm{~mm} / \mathrm{min}$ in the $0-0.25 \%$ strain range and was then increased to $10 \mathrm{~mm} / \mathrm{min}$ until specimen fracture occurred.

Dynamic mechanical thermal analysis (DMTA) in a bending configuration was carried out using Perkin Elmer equipment on the heat treated injection moulded specimens.
A frequency of $10 \mathrm{~Hz}$ was applied over the temperature range from -100 to $300{ }^{\circ} \mathrm{C}$, at a rate of $2{ }^{\circ} \mathrm{C} / \mathrm{min}$.

Field emission gun scanning electron microscopy (FEGSEM) was carried out on fracture surfaces of the injection moulded specimens after tensile testing. For imaging the samples were coated with a $20 \mathrm{~nm}$ layer of gold. In addition, transmission electron microscopy (TEM) analysis was performed on samples microtomed at room temperature.

The wear tests were performed according to ISO 7148-2/ section 5.2 with a ball-on-prism test system. Each material combination was tested three times for 60 hours. In all cases, the wear curves reached a steady state with a linear wear course. This part of the curve was fitted by a linear function of the type

$$
V=\dot{w}_{v / s} \cdot s+V_{i n},
$$

where $\dot{w}_{v / s}=\frac{d V}{d s}$ represents the steady state wear rate, $\mathrm{s}$ is the sliding distance and $\mathrm{V}_{\text {in }}$ is a measure for the running-in wear volume. Normalising $\dot{w}_{v / s}$ with respect to the normal load acting on a single specimen surface, $\mathrm{F}_{\mathrm{N}}$, results in the specific wear rate, $\dot{w}_{v / s, n}$, which was taken as a quantitative

measure for comparing the different material combinations. Light microscopic images were taken from the wear surfaces after the completion of the test.

\section{Results and Discussion}

Rheological tests were performed on PEEK compounds containing 0, 5, 10 and $15 \mathrm{wt} \%$ of carbon nanofibres. Figure 1 shows the shear viscosity of PEEK-CNF compounds as a function of the shear rate. Even though an increasing viscosity can be observed the higher the CNF loading, the observed shear thinning effect seems not to be strongly depending on the amount of the fibres. Tests for comparison on PEEKgraphite samples did show a similar behaviour in the high shear region suggesting processing parameters known from

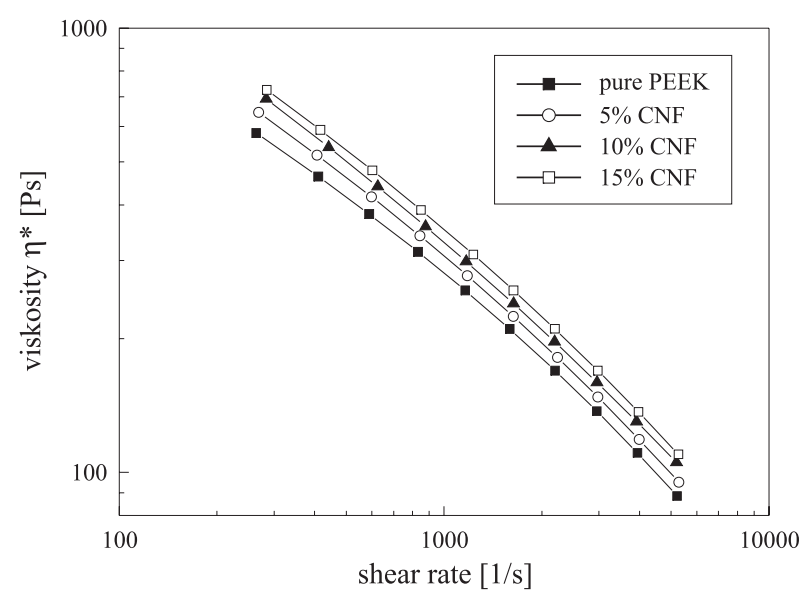

Figure 1. Shear viscosity of PEEK-CNF at $360^{\circ} \mathrm{C}$. 
graphite composites. Thus, most equipment suitable for PEEK processing methods such as injection moulding or extrusion processes can be used without modifications to handle PEEKCNF composites.

Isothermal DSC measurements revealed a two stage crystallization process indicating the same nucleating mechanisms and crystal grows kinetics for all CNF loadings. Heating ramp DSC measurements on the heat treated samples did reveal a constant degree of crystallinity of $30 \%$ normalized to the PEEK ensuring that any changes in mechanical properties are independent of morphology and thus are due to the fibres.

Representative stress-strain diagrams of the nanocomposites as a function of nanofibre loading are shown in Figure 2. At least five specimens were tested for each loading fraction and very small deviations in mechanical properties such as modulus, yield stress and strain to failure were observed, indicating a uniformity of the samples for all loading fractions. The actual volume fractions were determined from density measurements which revealed an effective CNF density of $2.0 \pm 0.04 \mathrm{~g} / \mathrm{cm}^{3}$. The tensile modulus of the pure PEEK matrix was found to be $4 \pm 0.1 \mathrm{GPa}$ and increased lineary by $40 \%$ to $5.6 \pm 0.2 \mathrm{GPa}$ for a loading fraction of $15 \mathrm{wt} \%$. There was also a linear increase in the stress at the yield point and composite strength. The strength increased from about $80 \mathrm{MPa}$ to about $120 \mathrm{MPa}$ for the 15 $\mathrm{wt} \%$ nanocomposite. A similar linear increase in modulus and strength was observed in a study of vapour-grown nanofibres dispersed in a polycarbonate matrix ${ }^{[13]}$. Nevertheless the yield strain of the PEEK composites was not affected. In addition, the strain to failure, about $22 \pm 1.2 \%$ for the pure matrix, was found not to decrease significantly for loading fractions up to $10 \mathrm{wt} \%$. In contrast, it dropped to about $4.5 \pm 0.3 \%$ for the $15 \mathrm{wt} \%$ nanofibre loading samples. This result is in contrast to previous studies in which the addition of nanofibres drastically reduced the impact properties of polycarbonate ${ }^{[14]}$ and the strain to failure of polypropylene ${ }^{[14]}$. Optical microscopy of the fractured PEEK composite samples revealed that the ability of the nanocomposites to contract and flow at the yield point

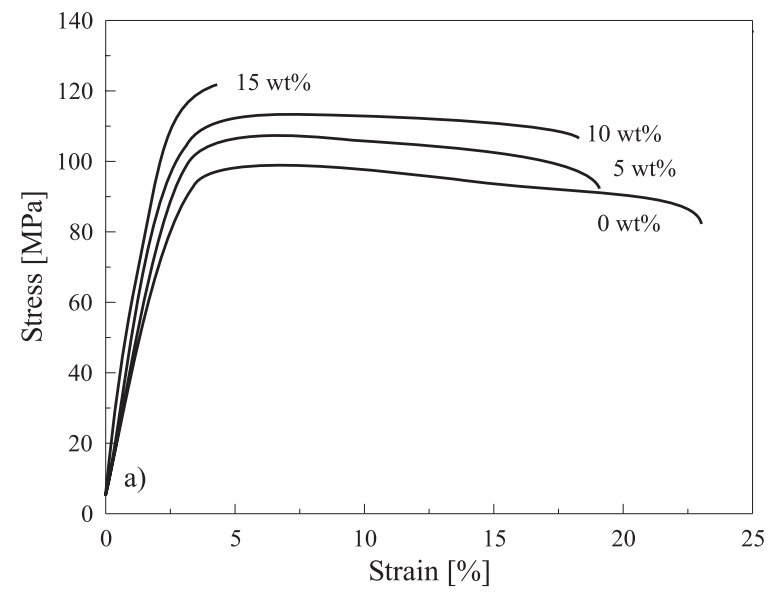

Figure 2. Stress strain curves of PEEK-CNF compounds (10mm/sec, RT).

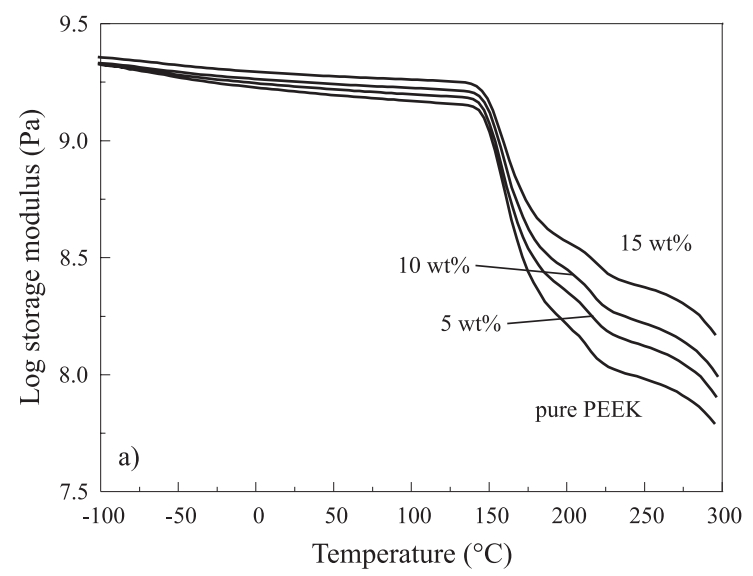

Figure 3. Dynamic mechanical analysis of PEEK nanocomposites as a function of temperature $-\log$ storage modulus.

decreased with increasing nanofibre content. This effect was most pronounced for the $15 \mathrm{wt} \%$ samples, which showed a brittle fracture behaviour.

The bending elastic modulus and damping properties of the nanocomposites were characterised by DMTA as a function of nanofibre loading and temperature. The CNF were aligned perpendicular to the direction of deformation. Figure 3 shows an increase in stiffness with nanofibres content both below and above the glass transition temperature $\mathrm{Tg}$ (around $1648^{\circ} \mathrm{C}$ ), the effect being more pronounced above the softening point of the matrix. The glass transition temperature itself was not affected as shown by the peak position of the tangent of the ratio of the loss to storage modulus ( $\tan \delta$ ), a measure of the damping within the system (Figure 4). The decreasing height of the peak with increasing nanofibre loading relates to the reduced fraction of polymer-matrix. The onset of the glass transition was not affected by the presence of the CNF which was also verified using DSC scans.

The fracture surfaces of the injection moulded nanocomposites specimens after tensile testing were examined in a FEGSEM to assess the dispersion and alignment of the nanofibres achieved during processing (Figure 5). In all the samples analysed, the nanofibres were found to be well

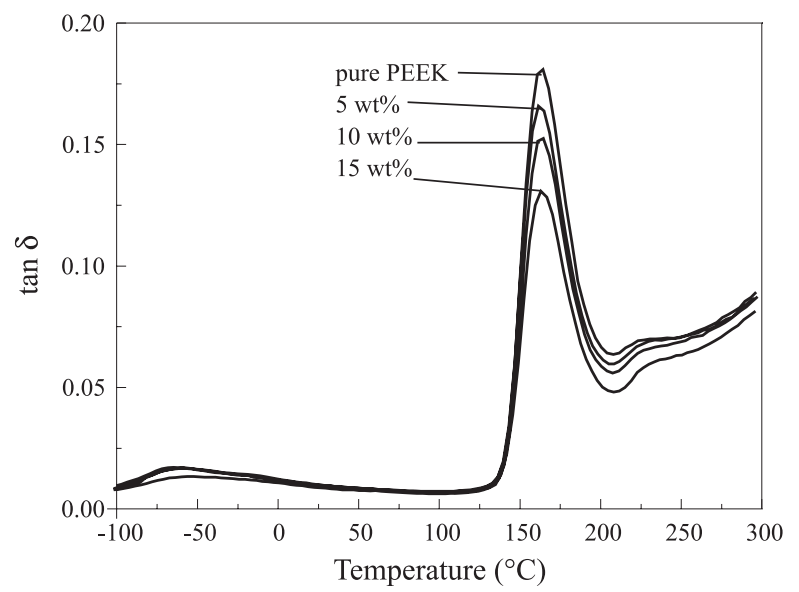

Figure 4. Dynamic mechanical analysis of PEEK nanocomposites as a function of temperature $-\tan \delta$. 


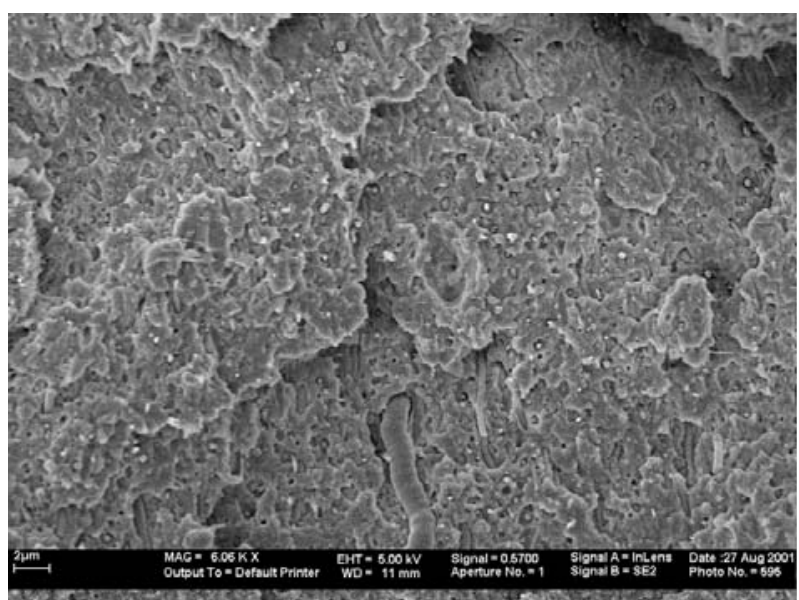

Figure 5. Scanning electron micrographs of fracture surfaces of nanocomposites containing $10 \mathrm{wt} \%$ vapour grown $\mathrm{CNF}$ after tensile testing.

dispersed and no voids could be observed. These conclusions are consistent with the uniformity observed in the tensile test data. In addition, the nanofibres were aligned with the direction of flow during processing. The degree of alignment was reduced in the core of the injection moulded samples where the shear flow is lower, an effect which is well known for injection moulded short-fibre composites ${ }^{[15]}$. For most nanofibres the pull-out length was less than $1 \mathrm{~mm}$, indicating fracture of individual nanofibres. Occasional pull-out lengths exceeding $2 \mathrm{~mm}$ can be seen, but the interfacial bonding between nanofibres and the PEEK appears to be strong. Further TEM analysis of the PEEK-CNF interface did support these results.

Results of wear tests for the three PEEK-CNF nanocomposites with carbon nanofibre contents of $0-15 \mathrm{wt} \%$ tested against the $100 \mathrm{Cr} 6$ steel are shown in Figure 6. As can be observed the CNF significantly reduce the specific wear rate of PEEK, however, it appears not to be strongly dependent on the CNF content within the range of 5 to $15 \%$ by weight. Nevertheless, there is a small optimum in the specific wear rate for a loading of $10 \mathrm{wt} \%$ of nanofibres. Such an optimum at about $10 \mathrm{wt} \%$ is also described in the literature for glass and

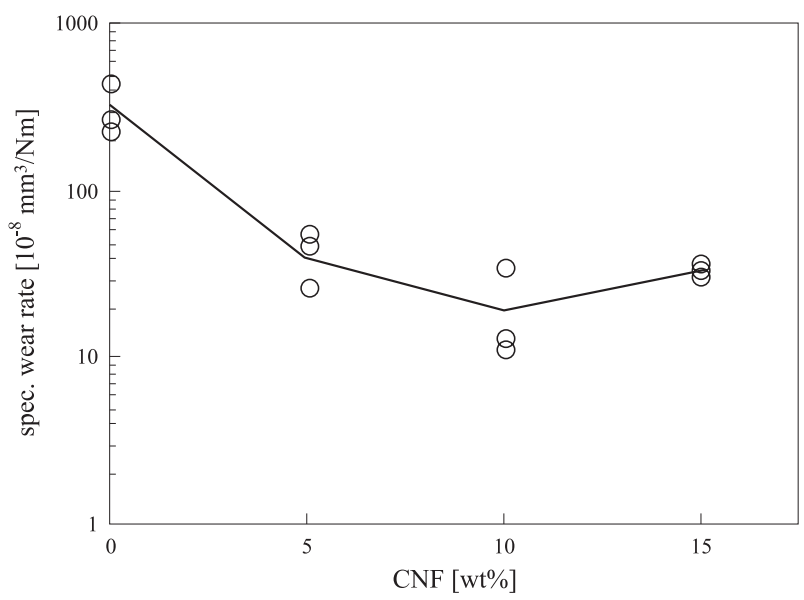

Figure 6: Semi-log plot of specific wear rate of PEEK-CNF compounds vs. $100 \mathrm{Cr} 6$ steel, as function of CNF content. carbon fibre-reinforced PEEK ${ }^{[16-18]}$. Possible reasons for the observed wear reduction are an increased shear strength of the CNF-nanocomposites compared to the pure PEEK, or a lubricating effect of the CNF wear debris. CNFs that are pulled out decompose to small graphitic debris particles which should essentially act as a lubricant similar to graphite flakes. Given the small size and the intrinsic graphitic structure of individual nanofibres, CNF pull-out is not expected to lead to any macroscopic surface roughness on either bearing surface, which was supported by light microscopic images.

\section{Conclusion}

PEEK-CNF composites can be successfully manufactured using standard processing technology. An increase in the melt strength without a significant increase of the shear viscosity at high shear rates can be observed. There is no nucleating effect due to the nanofibres; a two step crystal grows process can be observed for all PEEK samples for CNF loadings between 0 and $15 \mathrm{wt} \%$. After heat treatment the samples show a constant degree of crystallinity.

Due to a good dispersion and alignment of the CNFs within the injection moulded parts, coupled with a good interfacial bonding, the nanofibres act as a conventional reinforcement in the PEEK matrix not affecting the matrix ductility at loadings up to $10 \mathrm{wt} \%$. The stiffness increase is more pronounced above the glass transition of the polymer. The intrinsic stiffness of such vapour-grown nanofibres appears low (about half of glass fibres); nevertheless, they should be seen as suitable reinforcements for thermoplastic matrices, especially for delicate structures such as polymer fibres and films.

The wear rate of the macroscopic PEEK-CNF compounds is significantly reduced due to a lubricating effect of nanofibre wear debris. Like graphite, the CNFs seem to act as solid lubricants. Due to their small size, the roughening effect on the counterpart, which is observed for conventional fibre types, is minimised.

The small size of the CNFs coupled with their processability suggest new applications in the field of microstructured parts under mechanical or tribological loading conditions, which cannot be addressed using current composites based on macroscopic fillers.

\section{References}

1. Yakobson, B.I.; Brabec, C.J. \& Bernholc J. - Nanomechanics of carbon tubes: instabilities beyond linear response. Phys Rev Lett, 76, p.2511-4, (1996).

2. Ruoff, R.S. \& Lorents D.C. - Mechanical and thermal properties of carbon nanotubes. Carbon, 33, p.925-30, (1995).

3. Treacy, M.M.J.; Ebbesen, T.W.; Gibson, T.M. - Exceptionally high Young's modulus observed for individual carbon nanotubes. Nature, 381, p. 680-7, (1996). 
4. Wong, E.W.; Sheehan, P.E. \& Lieber, C.M. - Nanobeam mechanics: elasticity, strength, and toughness of nanorods and nanotubes. Science, 277, p.1971-5, (1997).

5. Yu, M.F.; Files, B.S.; Arepalli, S. \& Ruoff, R.S. - Tensile loading of ropes of single wall carbon nanotubes and their mechanical properties. Phys Rev Lett, 84, p.5552-5, (2000).

6. Yu, M.F.; Lourie, O.; Dyer, M.J.; Moloni, K.; Kelly, T.F. \& Ruoff, R.S. - Strength and breaking mechanism of multiwalled carbon nanotubes under tensile load. Science, 287, p.637-40, (2000).

7. Thostenson, E.T.; Ren, Z. \& Chou, T.W. - Advances in the science and technology of carbon nanotubes and their composites: a review. Compos Sci Technol, 61, p.1899-912, (2001).

8. Iijima, S. - Helical microtubules of graphitic carbon. Nature, 54, p.56-8, (1991).

9. Salvetat, J. P.; Kulik, A. J.; Bonard, J.M. \& Briggs, G. A. G.; Sto“ckli, T.; Me'te'nier, K.; Bonnamy, S.; Be'guin, F.; Burnham, N.A. \& Forro', L. - Elastic modulus of ordered and disordered multiwalled carbon nanotubes. Adv Mater, 11, p.161-5, (1999).

10. Wang, Q.; Xu, J.; Shen, W. and Liu, W. - An investigation of the friction and wear properties of nanometer $\mathrm{Si}_{3} \mathrm{~N}_{4}$ filled PEEK, Wear, 196, 82-86, (1996).
11. Wang, Q.; Xu, J. and Shen, W. - The friction and wear properties of nanometre $\mathrm{SiO}_{2}$ filled polyetheretherketone, Tribol. Int., 30, 193-197, (1997).

12. Dong, S. R.; Tu, J. P. and Zhang, X. P. - An investigation of the sliding wear behaviour of Cu-matrix composite reinforced by carbon nanotubes, Mat. Sci. Eng. A, 313, 83-87, (2001).

13. Carneiro, O. S.; Covas, J. A.; Bernado, C. A.; Caldeira, G.; Van Hattum, F. W. J.; Ting, J. M.; Alig, R. L. \& Lake, M. L. - Production and assessment of polycarbonate composites reinforced with vapour-grown carbon fibres. Compos Sci Technol, 58, p.401-7, (1998).

14. Lozano, K.; Barrera, E. V. - Nanofiber-reinforced thermoplastic composites. I. Thermoanalytical and mechanical analyses. J Appl Polym Sci, 79, p.125-33, (2001).

15. Chou TW. Microstructural design of fiber composites. Cambridge: Cambridge University Press; 1992.

16. Voß, H.; K. Friedrich, K. - On the Wear Behaviour of Short Fibre Reinforced PEEK Composites, Wear, 116, p.1-18, (1987)

17. Flöck, J.; Friedrich, K. \& Q. Yuan, Q. - On the friction and wear behaviour of PAN- and pitch-carbon fiber reinforced PEEK composites, Wear, 225-229, 304-311, (1999).

18. B. Mortimer, B. \& Lancaster, J. K. - Extending the life of aerospace dry bearings by the use of hard smooth counterparts, Wear, 121, 289-305, (1988). 